\title{
Warm Dark Matter Sterile Neutrinos in Electron Capture and Beta Decay Spectra
}

\author{
O. Moreno, ${ }^{1}$ E. Moya de Guerra, ${ }^{1}$ and M. Ramón Medrano ${ }^{2}$ \\ ${ }^{1}$ Departamento de Física Atómica, Molecular y Nuclear, Facultad de Ciencias Físicas, Universidad Complutense de Madrid, \\ 28040 Madrid, Spain \\ ${ }^{2}$ Departamento de Física Teórica I, Facultad de Ciencias Físicas, Universidad Complutense de Madrid, 28040 Madrid, Spain
}

Correspondence should be addressed to O. Moreno; osmoreno@mit.edu

Received 1 July 2016; Revised 21 September 2016; Accepted 3 October 2016

Academic Editor: Theocharis Kosmas

Copyright (C) 2016 O. Moreno et al. This is an open access article distributed under the Creative Commons Attribution License, which permits unrestricted use, distribution, and reproduction in any medium, provided the original work is properly cited. The publication of this article was funded by $\mathrm{SCOAP}^{3}$.

We briefly review the motivation to search for sterile neutrinos in the keV mass scale, as dark matter candidates, and the prospects to find them in beta decay or electron capture spectra, with a global perspective. We describe the fundamentals of the neutrino flavor-mass eigenstate mismatch that opens the possibility of detecting sterile neutrinos in such ordinary nuclear processes. Results are shown and discussed for the effect of heavy neutrino emission in electron capture in Holmium 163 and in two isotopes of Lead, 202 and 205, as well as in the beta decay of Tritium. We study the deexcitation spectrum in the considered cases of electron capture and the charged lepton spectrum in the case of Tritium beta decay. For each of these cases, we define ratios of integrated transition rates over different regions of the spectrum under study and give new results that may guide and facilitate the analysis of possible future measurements, paying particular attention to forbidden transitions in Lead isotopes.

\section{Introduction}

There is an inconsistency between the amount of matter inferred from gravitational effects and the one we see on observable scales. This fact leads to considering the existence of dark matter (DM). Evidence arises from astrophysical and cosmological probes [1-4], such as the kinematics of virially bound systems, rotation curves of spiral galaxies, strong and weak lensing, cosmic microwave background (CMB) information on matter density and geometry of the Universe, mass-to-light ratios in dwarf spheroidal galaxies (dSph), and large surveys to measure Universe structures. Because of the limits inferred from Big Bang nucleosynthesis, an important fraction of DM should be nonbaryonic. Although the nature of DM is still unknown, a popular hypothesis considers that DM consists of elementary particles $[3,4]$. DM is the main component of galaxies, being in average at least six times more abundant than baryonic matter (more than $81 \%$ of the matter in the Universe), but its nature is still unknown. DM self-interactions have been unobserved so far, and DM particle candidates are bound by gravitational interactions. Very distinct predictions for small scale structures in the Universe (below $100 \mathrm{kpc}$ ) are obtained by DM particles with different mass scales [5]. On the large mass scale side, WIMPs (weakly interacting massive particles) are popular candidates to $\mathrm{DM}$ that have masses of the order of $\mathrm{GeV}$ or even $\mathrm{TeV}$. This type of particles fall in the category of cold dark matter (CDM), which predicts, for small scales, too many galaxy satellites in the Milky Way and cusped profiles for the mass density of galaxies, contradicting present observational evidences. On the contrary, particles with mass in the keV scale, namely, warm dark matter (WDM) [6-15], are able to reproduce the number of observed satellite galaxies, as well as the cored profiles found in DM-dominated objects such as dwarf spheroidal galaxies.

Concerning the satellite problem, cosmic structures would form from the gravitational collapse of overdense regions in the DM primordial field. Free relativistic particles do not cluster, and structures at scales smaller than the particle free-streaming length $l_{\mathrm{fs}}$ are erased. The free-streaming 
length is the distance travelled freely by a relativistic particle after decoupling from the primordial plasma due to Universe expansion (approximately the distance travelled before the transition to nonrelativistic velocities). WDM particles (keV scale) give $l_{\mathrm{fs}} \sim 100 \mathrm{kpc}$, while CDM (GeV-TeV scale), which are heavier and slower than WDM, would give a $l_{\mathrm{fs}}$ a million times smaller and lead to the existence of a host of small scale structures [16]. On the galaxy density profiles $(\rho)$, CDM gives a steep cusp at the center $\left(\rho \sim r^{-1}\right)$ [17]. On the contrary, WDM gives a finite constant density core at the center $\left(\rho \sim \rho_{0}\right)$, in agreement with observations [18-24]. WDM quantum effects [25] could be important inside the galaxy core (below $100 \mathrm{pc}$ ), showing a fermionic nature for the DM particle through the manifestation of quantum nonvanishing pressure versus gravity.

Astrophysical observations from DM-dominated objects, as well as theoretical analysis, lead to a DM fermionic thermal particle with a mass around $2 \mathrm{keV}$ [26]. Chandra and XMMNewton detections in the X-ray spectra of the M31 galaxy and the Perseus cluster (both DM-dominated) seem to be consistent with sterile neutrinos of a few $\mathrm{keV}$. In particular, an unidentified $3.55 \mathrm{keV}$ line has been observed which seems compatible with the decay or annihilation of sterile relic neutrinos $[27,28]$ and that does not correspond to any known atomic emission. Although the interpretation of this line is stil subject to debate, it could be considered an indication of the decay of a $7.1 \mathrm{keV}$ nonthermal sterile neutrino $[29,30]$.

As it is well known, the Standard Model (SM) of elementary particles does not describe DM particles, nor does it provide a mass for active neutrinos $\left(v_{e}, v_{\mu}, v_{\tau}\right)$. In this model, neutrino eigenstates are left-handed, and they transform as doublets under the weak SU(2) gauge group. In extended SM models $[31,32]$, additional $\mathrm{SU}(3) \times \mathrm{SU}(2) \times \mathrm{U}(1)$ singlet righthanded neutrinos are introduced. Neutrino mass eigenstates will be linear combinations of the left and right-handed states, and mass matrix eigenvalues will split into lighter and heavier states (seesaw mechanism). The lighter mass eigenstates would be the main components of the active flavor eigenstates of the neutrinos, whereas the heavier ones would be dominant in sterile neutrino flavors [33]. Some extended SM models add three extra sterile neutrinos, one with a mass of the order of keV (see [34] and references therein). Neutrinos in the $\mathrm{keV}$ mass scale open the possibility to detect warm dark matter in nuclear electron capture and beta decay. Heavier sterile neutrinos cannot be produced in weak nuclear decays, and there are of course many other candidate particles for DM unconnected in principle to weak nuclear processes, such as the lightest supersymmetric particles $[35,36]$.

In this paper, we assume that a $\mathrm{keV}$ neutrino, a sound candidate for DM [37], is produced in ordinary weak nuclear processes, such as beta decay and electron capture, via neutrino mixing. Experiments searching for active neutrino masses, also look for sterile neutrinos in the $\mathrm{keV}$ range [38]: MARE [39-41] (that used Rhenium 187 beta decay and is not active anymore); KATRIN [42-46], PTOLEMY [47, 48], and Project8 [49] (using Tritium beta decay); and ECHo [50-52] and HOLMES [53] (using Holmium 163 electron capture). The mass of the neutrino (or antineutrino) emitted in a weak nuclear decay has an effect on the energy spectrum of the process, as was first shown by Enrico Fermi in the early 1930s [54, 55]. For the active neutrinos, this effect shows up at the endpoint of the spectrum, whereas for the sterile neutrinos considered here it may be expected to appear at a few keV below the endpoint. Clearly, in order to leave a fingerprint the sterile neutrino mass must be within the $Q$ window, that is, lower than the energy $Q$ available in the decay. The energy spectrum to be analyzed corresponds to the emitted charged lepton in the case of beta decay, namely, the spontaneous conversion of a neutron into a proton or vice versa with emission of the charged lepton and an antineutrino or neutrino. In the case of electron capture only a neutrino is emitted and no charged lepton comes out, so that the spectrum to be measured corresponds to the deexcitation of the daughter atom.

A summary of current experimental studies of neutrino properties in the frontiers of intensities and sensitivities is given in [62]. Experiments are under way to determine directly the active neutrino mass from neutrinoless double beta decay [63] as well as from single beta decay [42-48] and electron capture [50-53]. The last two types of experiments also provide a way to search for WDM sterile neutrinos in the measured spectra. In electron capture experiments, the spectrum collected in a calorimeter is directly linked to the excitation energies of the daughter atoms or molecules. In a calorimeter, the active source is embedded in the detector, which collects the energy of all the particles emitted in the deexcitation processes that take place in the source, except that of the neutrinos. In beta decay, the fact that the atoms or molecules may remain excited poses a challenge on the interpretation of the electron spectrum. Electron capture experiments take advantage of the larger statistics in the spectrum regions around the capture resonances. A limitation of electron capture measurements is that in a calorimeter, where the full energy range of the spectrum is measured, pile-up can be a problem that should be prevented by limiting the activity in the experimental setup. We refer always in this paper to Earth-based experiments where both the emission and the detection of particles take place in the laboratory. Other possible scenarios, like the search for sterile neutrinos in stellar matter (stellar beta decay and electron capture rates), are beyond the scope of this paper. The phase space for these rates increases manifold in stellar environment. For a reference on stellar weak rates, see [64, 65].

\section{Heavy Mass and Sterile Flavor in Neutrino States}

Already observed neutrino oscillations (see, e.g., [66-69]) among light active neutrinos are due to the fact that the neutrino flavor eigenstates and the mass eigenstates are not the same. Each flavor eigenstate, associated with a charged lepton, can be written as a combination of mass eigenstates and vice versa [70]. For instance, the neutrino flavor eigenstate emitted after electron capture, called electron neutrino $v_{e}$, can be written as a combination of the three light SM mass 
eigenstates and, hypothetically, of one or more extra, heavier mass eigenstates as [71-77]

$$
\left|\nu_{e}\right\rangle=U_{e 1}\left|\nu_{1}\right\rangle+U_{e 2}\left|\nu_{2}\right\rangle+U_{e 3}\left|v_{3}\right\rangle+\sum_{h} U_{e h}\left|v_{h}\right\rangle,
$$

where the subscript $h=\{4,5, \ldots\}$ stands for extra (heavier) mass eigenstates and the quantities $U$ belong to the unitary neutrino mixing matrix. The masses of the three light SM mass eigenstates are so close to each other that so far no measurement has been able to discern which of them has been emitted in a given process. As a result, the three are emitted as a coherent superposition, which is at the origin of the neutrino oscillation phenomenology. The phase of each mass eigenstate changes at a different rate while travelling, giving rise to a different superposition at each location that translates into a varying (oscillatory) probability of detection of a given flavor eigenstate.

To simplify things we will consider here the linear combination of light mass eigenstates as a single, effective neutrino mass eigenstate, called "light", with mass

$$
m_{l}=\bar{m}_{v_{e}}=\left(U_{e 1}^{2} m_{1}^{2}+U_{e 2}^{2} m_{2}^{2}+U_{e 3}^{2} m_{3}^{2}\right)^{1 / 2}
$$

and just one extra mass eigenstate, clearly heavier than the others, called "heavy" (with mass $m_{h}$, which we shall consider in the keV range):

$$
\left|\nu_{e}\right\rangle=\cos \zeta\left|\nu_{l}\right\rangle+\sin \zeta\left|\nu_{h}\right\rangle
$$

where the mixing amplitudes have been written in terms of a mixing angle $\zeta$ between the light and the heavy neutrino mass eigenstate. The other possible combination of these two mass eigenstates would be the sterile flavor eigenstate:

$$
\left|v_{s}\right\rangle=-\sin \zeta\left|v_{l}\right\rangle+\cos \zeta\left|v_{h}\right\rangle
$$

such that $\left\langle v_{e} \mid v_{s}\right\rangle=0$. For the sterile neutrinos that can be relevant as WDM, cosmological constraints based on the observed average dark matter density suggest that the value of the mixing angle could approximately be $\zeta=0.006^{\circ}$ [71-74], corresponding to a flavor-mass amplitude $U_{e h}=\sin \zeta \approx 10^{-4}$. Other recent cosmological constraints give values of $\sin ^{2}(2 \zeta)$ between $2 \cdot 10^{-11}$ and $2 \cdot 10^{-10}[27,28]$.

Sterile neutrino states with masses close to the ones of the active neutrinos can also have an impact on the patterns measured in oscillation experiments [78]. Heavier sterile neutrinos, as in particular in the $\mathrm{keV}$ scale, do not modify the oscillation patterns since they are not emitted coherently with the active neutrinos due to the large mass splitting.

The differential energy spectrum of a weak process where an electronic neutrino (or antineutrino) is emitted can therefore be decomposed in a term for light neutrino emission and another term for heavy neutrino emission as follows [75-77]:

$$
\frac{d \lambda}{d E}=\frac{d \lambda^{l}}{d E} \cos ^{2} \zeta+\frac{d \lambda^{h}}{d E} \sin ^{2} \zeta .
$$

This energy spectrum can be the one of the electron emitted in beta decay or the one of the daughter atom deexcitations in electron capture. The heavy mass eigenstate, if it exists, would be emitted independently of the other masses (noncoherently) as long as the energy resolution of the detector is better than the mass difference, $\Delta \epsilon<\left(m_{h}-\bar{m}_{e}\right)$. Its contribution to the measured spectrum, $d \lambda^{h} / d E$, would show up in the range where the collected energy is small enough for the heavy neutrino mass to have been produced, namely, when $0 \leq E \leq$ $\left(Q-m_{h}\right)$, where $Q$ is the difference between the masses of the initial and the final atoms when the reaction takes place in vacuum. At the edge of that region, at $E=Q-m_{h}$, a kink in the spectrum would be found with the size of the heavy neutrino contribution, namely, proportional to $\sin ^{2} \zeta \sim \zeta^{2}$ (the latter approximation valid for small mixing angles, as is the case in realistic scenarios).

\section{Theory of Electron Capture}

Let us consider the capture of an atomic electron by the nucleus $X(Z, N)$ to turn into the nucleus $Y(Z-1, N+1)$. The reactions involving the corresponding atoms (represented by the symbol of the nucleus within brackets) begin with electron capture:

$$
(X) \longrightarrow(Y)^{H}+v_{i}
$$

followed by deexcitation of the daughter atom. In (6), $v_{i}$ is a neutrino mass eigenstate $\left(\nu_{l}\right.$ or $\left.\nu_{h}\right)$ and the superscript $H$ accounts for the excited state of the atom $(Y)$ corresponding to an electron hole in the shell $H$, due to the electron capture from this shell in the parent atom. The deexcitation of the daughter atom after electron capture can happen either through emission of a photon (X-ray emission):

$$
(Y)^{H} \longrightarrow(Y)+\gamma
$$

or through emission of electrons (Auger or Coster-Kronig process) and photons:

$$
(Y)^{H} \longrightarrow\left(Y^{+}\right)^{H^{\prime}, H^{\prime \prime}}+e^{-} \longrightarrow\left(Y^{+}\right)+\gamma+e^{-},
$$

where $H^{\prime}$ and $H^{\prime \prime}$ represent holes in the electron shells of the ion $\left(Y^{+}\right)$.

The energies carried by the emitted photons and electrons are deposited in the calorimeter that surrounds the source and that measures the full energy spectrum of these particles, provided that the corresponding deexcitation lifetimes are much smaller than the detector time response [79]. The process in (7) and (8) yields the same calorimeter spectrum; that is, the peaks appear at the same energy values corresponding to the excitation energies, $E_{H}$, of the excited daughter atom $(Y)^{H}$. The excitation energy is the difference between the binding energies of the captured electron shell and the additional electron in the outermost shell. The former refers to the daughter atom, whereas the latter refers to the parent: $E_{H} \approx\left|B_{H}^{(Y)}\right|-\left|B_{\text {out }}^{(X)}\right|$. The reason is that for the shells above the vacancy the effective charge is closer to the one in the parent atom (there is one proton less in the nucleus but also one electron less in an inner shell) and therefore its binding energies should be used [80]. 
An alternative capture process to the one in (6) involves the instantaneous formation of a second hole $H^{\prime}$ due to the mismatch between the spectator electron wave functions in the parent and in the daughter atom. Whereas the hole $H$ is left by the captured electron and therefore fulfils the energy and angular momentum conditions for such process, the extra hole $H^{\prime}$ has a different origin, namely, the above mentioned incomplete overlap of electron wave functions, which may occur in many electron shells. The electron leaving the extra hole may have been "shaken up" (excited) to an unoccupied orbital in the daughter atom:

$$
(X) \longrightarrow(Y)^{H, H^{\prime}}+v_{i},
$$

or it may have been "shaken off" to the continuum (ejected):

$$
(X) \longrightarrow\left(Y^{+}\right)^{H, H^{\prime}}+e^{-}+v_{i} .
$$

In a shake-up process, the deexcitation of the final atom contributes to the calorimeter energy with a peak at an energy approximately equal to the sum of the binding energies of the $H$ and $H^{\prime}$ electrons, $E_{H, H^{\prime}} \approx\left|B_{H}^{(Y)}\right|+\left|B_{H^{\prime}}^{(X)}\right|-\left|B_{\text {out }}^{(X)}\right|$. Thus, satellite peaks show up located after the single-hole peak at $E_{H}$. In the case of a shake-off, the calorimeter energy is the combination of $E_{H, H^{\prime}}$ and the electron kinetic energy, the latter showing a continuous distribution as corresponds to the three-body decay of (10).

It is important to notice that some electron emission processes of the type

$$
(X) \longrightarrow\left(Y^{+}\right)^{H^{\prime}, H^{\prime \prime}}+e^{-}+v_{i},
$$

where none of the holes in the daughter atom correspond to the captured electron $H$, are quantum mechanically indistinguishable $[81,82]$ from the three-step process in (6) and (8), and therefore they contribute to the same onehole peaks at $E_{H}$. The contributions to the spectrum of the two-hole excitations that are actually distinct from the onehole excitations ((9) and (10)) have been a subject of recent studies devoted to search for the electron neutrino mass and are therefore focused on the endpoint of the spectrum (see, e,g., [80-83]). For heavy neutrino searches, a similar analysis should be performed at other regions of the energy spectrum, not just at the endpoint. In this paper, we restrict the calculations to the one-hole states.

The density of final states of the emitted neutrino in electron capture is proportional to

$$
\rho=\rho_{\nu}\left(E_{v}\right) \propto p_{\nu} E_{v}=\left(E_{\nu}^{2}-m_{\nu}^{2}\right)^{1 / 2} E_{\nu}
$$

where $E_{v}, p_{v}$, and $m_{v}$ are the neutrino total energy, momentum, and mass, respectively. On the other hand, the probability of capture of a bound electron follows a Breit-Wigner distribution of width $\Gamma_{x}$ and peaks at the energy $E_{x}$ :

$$
P(E)=\frac{\Gamma_{x} / 2 \pi}{\left(E-E_{x}\right)^{2}+\Gamma_{x}^{2} / 4} .
$$

Using Fermi's Golden Rule, the differential reaction rate with respect to the neutrino energy yields

$$
\begin{aligned}
\frac{d \lambda}{d E_{v}}= & K_{\mathrm{EC}}\left(E_{\nu}^{2}-m_{\nu}^{2}\right)^{1 / 2} E_{\nu} \sum_{H} W_{H}^{(v)} \\
& \cdot \frac{\Gamma_{H} / 2 \pi}{\left[E_{v}-\left(Q-E_{H}\right)\right]^{2}+\Gamma_{H}^{2} / 4}
\end{aligned}
$$

where $K_{\mathrm{EC}}$ contains, among other factors, the weak interaction coupling constant and the nuclear matrix element. The factor $W_{H}^{(\nu)}$ is the squared leptonic matrix element for a given hole state $H$.

We write $W_{H}^{(\nu)}=C_{H} S_{H}^{(v)}$ to have a general expression for allowed and forbidden transitions. The factor $C_{H}$ is the squared amplitude of the bound-state electron radial wave function at the nuclear interior, containing also the squared overlap between the initial and the final atom orbital wave functions and the effect of electron exchange (as defined in Appendix F-2 of [56]). The cases discussed in the next sections involve allowed Gamow-Teller transitions ( ${ }^{163} \mathrm{Ho}(7 /$ $\left.\left.2^{-}\right) \rightarrow{ }^{163} \mathrm{Dy}\left(5 / 2^{-}\right)\right)$and first forbidden Gamow-Teller transitions $\left({ }^{202} \mathrm{~Pb}\left(0^{+}\right) \rightarrow{ }^{202} \mathrm{Tl}\left(2^{-}\right)\right.$and ${ }^{205} \mathrm{~Pb}\left(5 / 2^{-}\right) \rightarrow{ }^{205} \mathrm{Tl}(1 /$ $\left.2^{+}\right)$). In the first case, the factor $S_{H}^{(\nu)}=1$ for all $H$ states. In the second case, the factor $S_{H}^{(\nu)}=1$ for electrons captured with orbital angular momentum $l=1$ (and neutrinos emitted with $l=0)$ and $S_{H}^{(v)}=p_{\nu}^{2}$ for electrons captured with orbital angular momentum $l=0$ (and neutrinos emitted with $l=$ 1). The reason for this is that, due to conservation of total angular momentum, first forbidden transitions have $L=1$ for the leptonic pair. Hence, there is a linear momentum dependence in the leptonic matrix element corresponding to the lepton that carries the angular momentum in each specific capture. The corresponding $p_{e}^{2}$ factor for the $l=1$ electrons is embedded in the $C_{H}$ factor [56].

Equation (14) includes every possible orbital electron capture, the probability of each peaking at $E_{v}=Q-E_{H}$, as dictated by energy conservation, where $E_{H}$ is the excitation energy of the final atom due to the electron hole $H$ resulting from capture. The energy collected by a calorimeter from the atomic deexcitations is $E_{c}=Q-E_{\gamma}$, namely, all the available energy except for the one carried away by the neutrino. The differential rate can be written in terms of the calorimeter energy as

$$
\begin{aligned}
\frac{d \lambda}{d E_{c}}= & K_{\mathrm{EC}}\left[\left(Q-E_{c}\right)^{2}-m_{\nu}^{2}\right]^{1 / 2}\left(Q-E_{c}\right) \sum_{H} C_{H} S_{H}^{(\nu)} \\
& \cdot \frac{\Gamma_{H} / 2 \pi}{\left(E_{c}-E_{H}\right)^{2}+\Gamma_{H}^{2} / 4} .
\end{aligned}
$$

In the next section, we shall also consider integrated rates over particular energy ranges, which can be calculated numerically from (15). A convenient compact analytical expression of the integrated rate can be obtained from (15) by replacing the Breit-Wigner distributions by Dirac deltas. 
With this approximation, the integral of the differential rate can be written as

$$
\lambda=K_{\mathrm{EC}} \sum_{H} C_{H} S_{H}^{(\nu)}\left[\left(Q-E_{H}\right)^{2}-m_{\nu}^{2}\right]^{1 / 2}\left(Q-E_{H}\right) .
$$

In this theoretical model, we have neglected two-hole peaks, deexcitations through virtual intermediate states, and interferences between deexcitation channels. The theoretical calorimeter spectrum is thus a single-hole approximation that assumes full collection of de-excitation energy by the calorimeter and no pile-up.

\section{Sterile Neutrino Effect in the Spectrum}

Due to the smallness of the mixing angle, it is useful to consider ratios between rates that emphasize the contribution of the heavy neutrino. In [84-86], for the case of beta decay, a ratio was considered between the contributions of the heavy and the light neutrino to the differential rates, as we shall see in Section 6. For the case of electron capture, the differential decay rates contain many peaks and it is more convenient to consider integrated decay rates over specific energy ranges, as discussed in [87]. In either case (beta decay and electron capture), this amounts to consider that the detector collects events within energy ranges $E_{i} \pm \Delta$ and $E_{j} \pm \Delta$ in two regions of the spectrum, such that the mass of the hypothetical heavy neutrino lies in between, namely, $E_{i}+\Delta<m_{h}<E_{j}-\Delta$, with $E_{j}<Q-\Delta$. A ratio $R$ between the number of collected events in both regions is then performed, which corresponds to the theoretical expression:

$$
R=\frac{\Lambda_{i}}{\Lambda_{j}}=\frac{\kappa_{i}^{l}+\tan ^{2} \zeta \kappa_{i}^{h}}{\kappa_{j}^{l}}
$$

where $\Lambda_{i}=\cos ^{2} \zeta \kappa_{i}^{l}+\sin ^{2} \zeta \kappa_{i}^{h}$ (in the region where both $l$ and $h$ mass eigenstates contribute) and $\Lambda_{j}=\cos ^{2} \zeta \kappa_{i}^{l}$ (in the region where $h$ is not energetically allowed). The integrals $\kappa$ are defined as

$$
\kappa_{i, j}^{\nu}=\int_{E_{i, j}-\Delta}^{E_{i, j}+\Delta} \frac{d \lambda}{d E_{c}}\left(m_{\nu}\right) d E_{c} .
$$

In electron capture, the energies $i$ and $j$ can be selected as the energies of two peaks and the integration intervals can be chosen as the width of the capture peaks. If the peaks are approximated by delta functions, the integrals (using $E_{H}=E_{i}$ and $\Delta \rightarrow 0$ ) can be written as

$$
\kappa_{i ; r}^{\nu \mathrm{EC}}=K_{\mathrm{EC}} C_{i ; r} S_{i ; r}^{(v)}\left(Q_{r}-E_{i}\right)^{2}\left[1-\left(\frac{m_{v}}{Q_{r}-E_{i}}\right)^{2}\right]^{1 / 2},
$$

where $Q_{r}$ is the atomic mass difference in a given isotope $r$, $E_{i}$ is the energy position of a given peak $i$ in the calorimeter spectrum, $m_{v}$ is $m_{l} \approx 0$, the mass of a light neutrino, or $m_{h}$, the mass of a heavy neutrino, and $S_{i ; r}^{(v)}$ contains the neutrino momentum dependence for the peak $i$ coming from the leptonic matrix element squared. As explained in the previous section, for allowed transitions, $S_{i ; r}^{(\nu)}=1$. For first forbidden transitions, some of the capture peaks have $S_{i ; r}^{(\nu)}=p_{v i ; r}^{2}$ for the $i$ peaks corresponding to $s_{1 / 2}$ shells (as well as for those corresponding to $p_{1 / 2}$ shells that contribute through their admixtures with the $l=0$ orbital due to relativistic corrections). The other peaks have $S_{i ; r}^{(v)}=1$, where $i$ corresponds to $p_{3 / 2}$ shells (and $d_{3 / 2}$ shells through their admixtures with the $l=1$ orbital due to relativistic corrections).

For electron capture, it is convenient to define a ratio similar to the one in (17) but where the numerator and the denominator are themselves ratios of numbers of events within the same peak but for different isotopes of the same element, $r$ and $s$, so that some atomic corrections cancel out [87]

$$
R^{\prime}=\frac{\Lambda_{i ; r} / \Lambda_{i ; s}}{\Lambda_{j ; r} / \Lambda_{j ; s}}=\left(R_{i j ; r s}^{l}\right)^{2(\gamma+1)}\left(\frac{1+\omega_{i ; r}^{2 \gamma+1} \tan ^{2} \zeta}{1+\omega_{i ; s}^{2 \gamma+1} \tan ^{2} \zeta}\right),
$$

where one should notice that the factors $C_{i}$ and $C_{j}$ cancel out in addition to the factor $K_{\mathrm{EC}}$ which cancels out in both ratios ( $R$ and $R^{\prime}$ ). The factors in (20) have very simple analytical forms when one uses (19)

$$
\begin{aligned}
R_{i j ; r s}^{l} & =\frac{\left(Q_{r}-E_{i}\right)\left(Q_{s}-E_{j}\right)}{\left(Q_{r}-E_{j}\right)\left(Q_{s}-E_{i}\right)}, \\
\omega_{i ; r(s)} & =\left[1-\left(\frac{m_{h}}{Q_{r(s)}-E_{i}}\right)^{2}\right]^{1 / 2},
\end{aligned}
$$

where $\gamma$ depends on the angular momenta of each lepton in a given $\Delta J^{\pi}$ nuclear transition. For instance, for allowed decays, $\gamma=0$, whereas for first forbidden decays, $\gamma=1$ for $s_{1 / 2}$ and $p_{1 / 2}$ peaks or $\gamma=0$ for $p_{3 / 2}$ and $d_{3 / 2}$ peaks. In the expressions above, we have assumed that both peaks $i$ and $j$ are of the same type; namely, $\gamma_{i}=\gamma_{j}=\gamma$.

By measuring the ratio in (20) the mixing angle can then be obtained as

$$
\zeta=\arctan \left[\frac{\left(R_{i j ; r s}^{l}\right)^{2(\gamma+1)}-R_{\exp }^{\prime}}{R_{\exp }^{\prime} \omega_{i ; s}^{2 \gamma+1}-\left(R_{i j ; r s}^{l}\right)^{2(\gamma+1)} \omega_{i ; r}^{2 \gamma+1}}\right]^{1 / 2},
$$

where the theoretical values of $R_{i j ; r s}^{l}, \omega_{i ; r}$, and $\omega_{i ; s}$ require an accurate experimental knowledge of the position of the selected peaks and, more importantly, of the atomic mass differences $Q_{r}$ and $Q_{s}$. In addition, one has to consider fixed, initially unknown, values of the mass of the heavy neutrino $m_{h}$ that is searched for or otherwise be content with the generation of exclusion plots in case of no effect observation.

In summary, we consider here two types of ratios that can be useful in the search for a signal of $\mathrm{keV}$ sterile neutrinos in electron capture: (1) the ratio between the intensity of two peaks in the electron capture spectrum of a given nucleus, as for the case of ${ }^{163}$ Ho discussed in Section 5. (2) The case in which two isotopes of a given element undergo electron capture where one can use the ratio defined in (20), as is the case of Lead isotopes discussed in Section 5. For the 


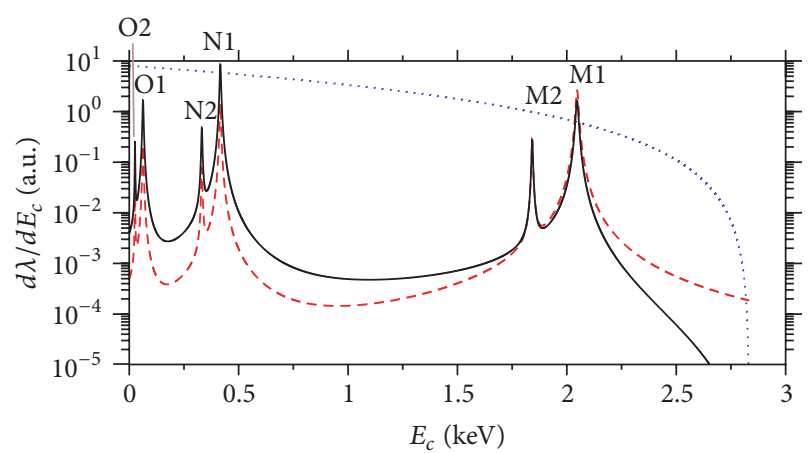

Neutrino phase space

- - - Atomic deexcitation - Total

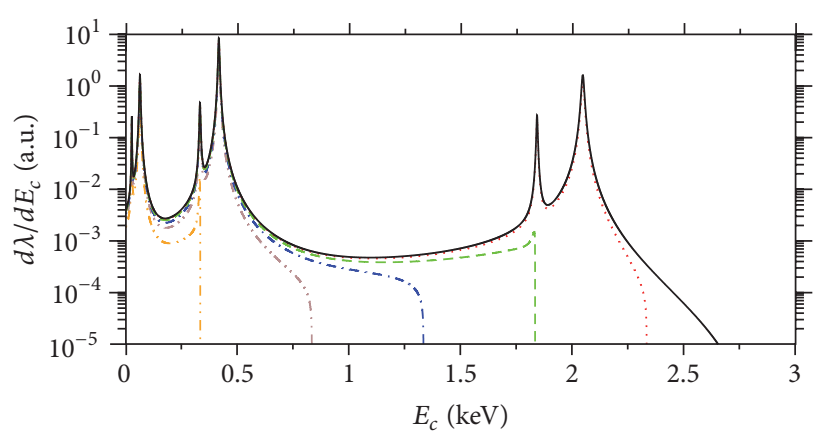

$$
\begin{aligned}
-\cdots m & =2.5 \mathrm{keV} & ---m & =1 \mathrm{keV} \\
\ldots m & =2 \mathrm{keV} & \ldots \ldots m & =0.5 \mathrm{keV} \\
-\cdots m & =1.5 \mathrm{keV} & - & -m=0
\end{aligned}
$$

(a)

(b)

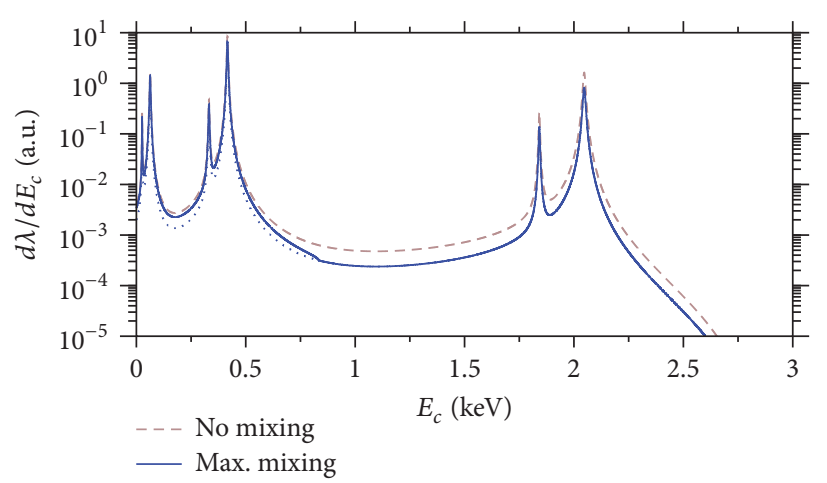

(c)

FIGURE 1: Calorimeter spectrum after electron capture in ${ }^{163}$ Ho. (a) Full spectrum (solid curve) and contributions: emitted neutrino phase space (dotted curve) and daughter atom deexcitation (dashed curve). (b) Full spectrum for different neutrino masses: 0 , 0.5, 1, 1.5, 2, and $2.5 \mathrm{keV}$. (c) full spectrum for light $\left(m_{l} \approx 0 \mathrm{keV}\right)$ - heavy $\left(m_{h}=2 \mathrm{keV}\right)$ neutrino mixing using a maximal mixing angle $\zeta=45^{\circ}$ for illustration, with (solid curve) and without (dotted curve) the heavy neutrino contribution. For comparison, the spectrum without heavy neutrino mixing (dashed curve) is also shown. We use the maximal unrealistic value $\zeta=45^{\circ}$ to make the effect visible in the scale of the figure. Realistic values of $\zeta<0.01^{\circ}$ result in a reduction of the difference between the dashed and the solid lines in (c) by more than seven orders of magnitude.

beta decay case, in Section 6 we consider the ratio between the differential rates, but the ratios between integrated rates are also useful and may be more realistic for comparison to experiments.

\section{Results for Electron Capture Spectra}

Let us first consider the capture of an atomic electron by the nucleus Holmium $163\left({ }^{163} \mathrm{Ho} ; Z=67, N=96\right)$ to turn into Dysprosium 163 ( $\left.{ }^{163} \mathrm{Dy} ; Z=66, N=97\right)$, with ground state spins and parities $J^{\pi}=7 / 2^{-}$and $J^{\pi}=5 / 2^{-}$, respectively. It is an allowed unique Gamow-Teller transition $(\Delta J=1$ and no parity change, the leptons carry no orbital angular momentum, $\Delta L=0$, and couple to spin $S=1$ ), with atomic mass difference $Q=2.833 \pm 0.030 \pm 0.015 \mathrm{keV}$ [88], which is being currently measured at the Electron Capture Holmium (ECHo) experiment [50-52]. Only electrons with principal quantum number $n$ larger than 2 have a binding energy lower than the $Q$-value and can therefore be captured. In addition, being an allowed decay, only electrons from $s_{1 / 2}$ and $p_{1 / 2}$ orbitals can be captured, the latter through admixtures with the $l=0$ orbital due to relativistic corrections. Thus, capture from the orbitals M1, M2, N1, N2, O1, O2, and P1 (in spectroscopic notation) is possible [80, 89-91].

In Figure 1, we show the differential electron capture rate in ${ }^{163} \mathrm{Ho}$ as a function of the calorimeter energy, $E_{c}=Q-E_{v}$. In Table 1, we give theoretical values of the strengths and widths of each capture peak and experimental values of the electron binding energy again for each capture peak. The strengths $C_{H}$ are obtained in $[56,57]$ from relativistic Hartree-Fock calculations of the electron wave functions at the nuclear site, accounting for exchange and overlap contributions. In the upper plot the emitted neutrino phase space contribution and the daughter atom deexcitation contribution are plotted separately, together with the product of both, which is the full differential electron capture rate. In the middle plot we show the full differential rate for different masses of the emitted neutrino: $m_{v} \approx 0$ (realistic) and $m_{v} \approx$ $0.5,1,1.5,2$, and $2.5 \mathrm{keV}$ (unrealistic). As can be seen in the plot, each curve ends at $Q-m_{v}$. Finally, the lower plot shows the spectrum resulting from the mixing of a light neutrino 
TABLE 1: Atomic parameters for electron capture in Holmium from shells $\mathrm{M} 1$ to $\mathrm{O} 2$. The values of $C_{H}[56,57]$ correspond to the atomic shells in Holmium, while the orbital electron widths $\Gamma_{H}$ [58] and binding energies $B_{H}(<Q)$ [59-61] correspond to the atomic shells in Dysprosium.

\begin{tabular}{lcccccc}
\hline & $\mathrm{M} 1$ & $\mathrm{M} 2$ & $\mathrm{~N} 1$ & $\mathrm{~N} 2$ & $\mathrm{O} 1$ & 0.001708 \\
\hline$C_{H}$ & 0.05377 & 0.002605 & 0.01373 & 0.0005891 & 0.0001 \\
$\Gamma_{H}[\mathrm{keV}]$ & 0.013 & 0.006 & 0.006 & 0.005 & 0.005 & 0.002 \\
$B_{H}[\mathrm{keV}]$ & 2.047 & 1.842 & 0.416 & 0.332 & 0.063 \\
\hline
\end{tabular}

TABLE 2: Same as Table 1 (data from the same references) but for electron capture in Lead going to Thallium. The upper table summarizes the values of the relevant parameters for $s_{1 / 2}$ and $p_{1 / 2}$ atomic shells, while the lower table shows the values for $p_{3 / 2}$ and $d_{3 / 2}$ atomic shells. In the latter case, $C_{H}$ includes an extra factor $p_{e}^{2}$.

\begin{tabular}{lcccccccc}
\hline & L1 & L2 & M1 & M2 & N1 & N2 & O1 \\
\hline$C_{H}$ & 0.8781 & 0.06647 & 0.2125 & 0.01759 & 0.05849 & 0.004431 & 0.01016 & 0.0008052 \\
$\Gamma_{H}[\mathrm{keV}]$ & 0.011 & 0.006 & 0.015 & 0.010 & 0.009 & 0.007 & - & - \\
$E_{H}[\mathrm{keV}]$ & 15.346 & 14.697 & 3.703 & 3.415 & 0.845 & 0.720 & 0.136 & 0.099 \\
\hline & $\mathrm{L} 3$ & $\mathrm{M} 3$ & $\mathrm{M} 4$ & $\mathrm{~N} 3$ & $\mathrm{~N} 4$ & $\mathrm{O} 3$ & $\mathrm{O} 4$ & 1.500 \\
\hline$C_{H}\left[\mathrm{keV}^{2}\right]$ & 18978.193 & 5366.014 & 45.383 & 1363.307 & 12.607 & 237.280 & 0.001 \\
$\Gamma_{H}[\mathrm{keV}]$ & 0.006 & 0.009 & 0.002 & 0.006 & 0.004 & 0.001 & 0.015 \\
$B_{H}[\mathrm{keV}]$ & 12.657 & 2.956 & 2.484 & 0.608 & 0.406 & 0.072 & \\
\hline
\end{tabular}

mass eigenstate, $m_{l} \approx 0$, with a heavy mass eigenstate $m_{h} \approx$ $2 \mathrm{keV}$ (solid curve) as in (5). Although the spectrum with mixing is realistic in the sense that it is the result expected if a heavy mass eigenstate exists, it is unrealistic in the degree of mixing shown in the figure, which has been maximized here for the sake of visibility of the "kink" in the scale of the figure: $50 \%$ light neutrino and 50\% heavy neutrino, corresponding to a mixing angle $\zeta=45^{\circ}$. The "kink" can be seen in this curve at $E_{c}=Q-m_{h}=0.833 \mathrm{keV}$. Above this calorimeter energy, the heavy mass eigenstate cannot be produced due to energy conservation. For comparison, the spectrum for $m_{v} \approx 0$ with no mixing with heavy states is shown in the dashed curve.

Another example of electron capture under study here is that of the Lead isotopes $202\left({ }^{202} \mathrm{~Pb} ; Z=82, N=120\right)$ and $205\left({ }^{205} \mathrm{~Pb} ; Z=82, N=123\right)$ going to the Thallium isotopes $202\left({ }^{202} \mathrm{Tl} ; Z=81, N=121\right)$ and $205\left({ }^{205} \mathrm{Tl}\right.$; $Z=81, N=124)$, respectively, where the process is in both cases a first forbidden Gamow-Teller unique transition, with $0^{+} \rightarrow 2^{-}$for ${ }^{202} \mathrm{~Pb}$ and $5 / 2^{-} \rightarrow 1 / 2^{+}$for ${ }^{205} \mathrm{~Pb}(\Delta J=2$ and parity change, the leptons carry $\Delta L=1$ and couple to $S=1$ ). The atomic mass differences obtained from the data in [92] are $Q=46 \pm 14 \mathrm{keV}$ and $Q=50.6 \pm 1.8 \mathrm{keV}$, respectively. According to the explanations given in Section 3, captures from orbitals L1, L2, M1, M2, N1, N2, O1, and O2 contain an extra neutrino momentum dependence $S^{(\nu)}=p_{v}^{2}$ that modifies the calorimeter spectrum and corresponds to $\gamma=$ 1. On the other hand, captures from orbitals L3, M3, M4, $\mathrm{N} 3, \mathrm{~N} 4, \mathrm{O} 3$, and $\mathrm{O} 4$, which correspond to $\gamma=0$, contain an extra electron momentum dependence $p_{e}^{2}$ which, being fixed in bound electrons, is included in the values of $C_{H}$. They are given in Table 2 together with the widths and the experimental electron binding energies in the daughter atom, Thallium.

Figure 2 shows the capture differential rate in ${ }^{205} \mathrm{~Pb}$ (dark curves) and in ${ }^{202} \mathrm{~Pb}$ (light curves). Position, width, and strength of the capture peaks are assumed to be the same in both isotopes, but the spectra are different because of the different $Q$-values. Solid curves are for light neutrino emission, $m_{v} \approx 0$, and dashed lines are for heavy neutrino emission with $m_{v}=40 \mathrm{keV}$ (results are given separately for each mass, before mixing). Due to the large $Q$-values, electron capture in Lead isotopes allows us to explore this mass value, although it may be somewhat larger than the expected value from cosmological reasons. The analysis of the ratio in (20) can be computed, for example, using the capture peaks L3 at $E_{c}=12.657 \mathrm{keV}$ and $\mathrm{M} 3$ at $E_{c}=2.956 \mathrm{keV}$, both being of the $\gamma=0$ type. For $m_{h}=40 \mathrm{keV}$ one would obtain $R_{M 3 L 3 ; 202205}^{1}=1.037, \omega_{M 3 ; 202}=0.369, \omega_{M 3 ; 205}=0.543$. These results should be introduced in (22), together with the experimental ratio $R_{\text {exp }}^{\prime}$, to obtain the value of the mixing angle $\zeta$.

It is important to remark that the theoretical quantities $R^{l}$ and $\omega$ computed as described above contain several approximations. They correspond to Dirac-delta peaks, ignoring the actual shapes and widths, and thus they just refer to one single capture peak, neglecting the possible effect of the tails of nearby one-hole peaks. Moreover, the influence of twohole peaks close to the main ones has also been neglected. Other effects that have not been taken into account but whose influence is expected to be very small are multihole (more than two) peaks, virtual intermediate states (influence of transitions through atomic shells not energetically accessible), or interference between atomic transitions resulting from addition of amplitudes instead of intensities [81, 82]. The accurate experimental determination of the ratio $R_{\exp }^{\prime}$ entails its own difficulties, among them the possible existence of metastable atomic states whose delayed de-excitations fail to contribute to the collected spectrum, and the variety of chemical environments resulting in a complex mixture of $Q$ values. 


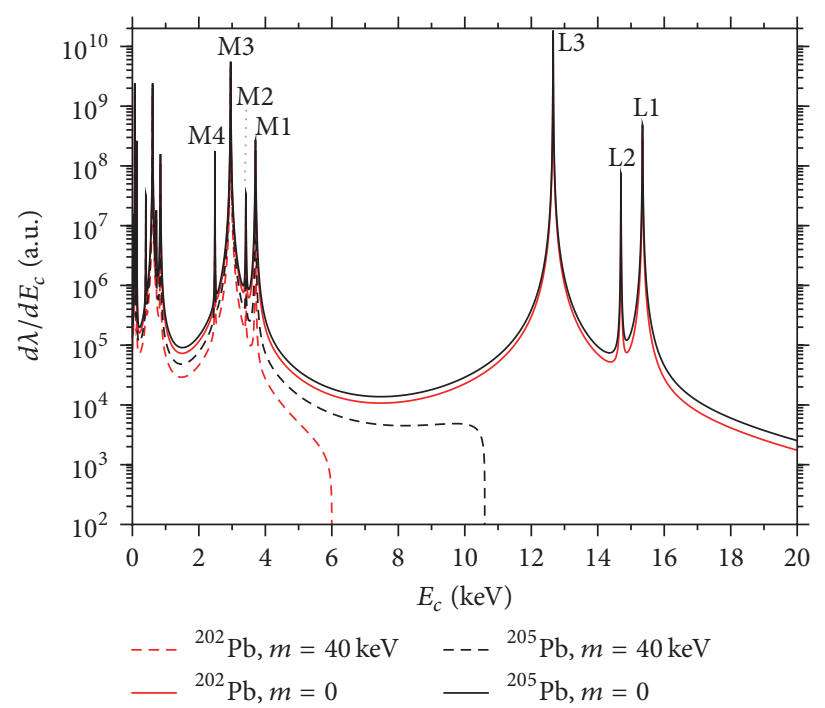

(a)

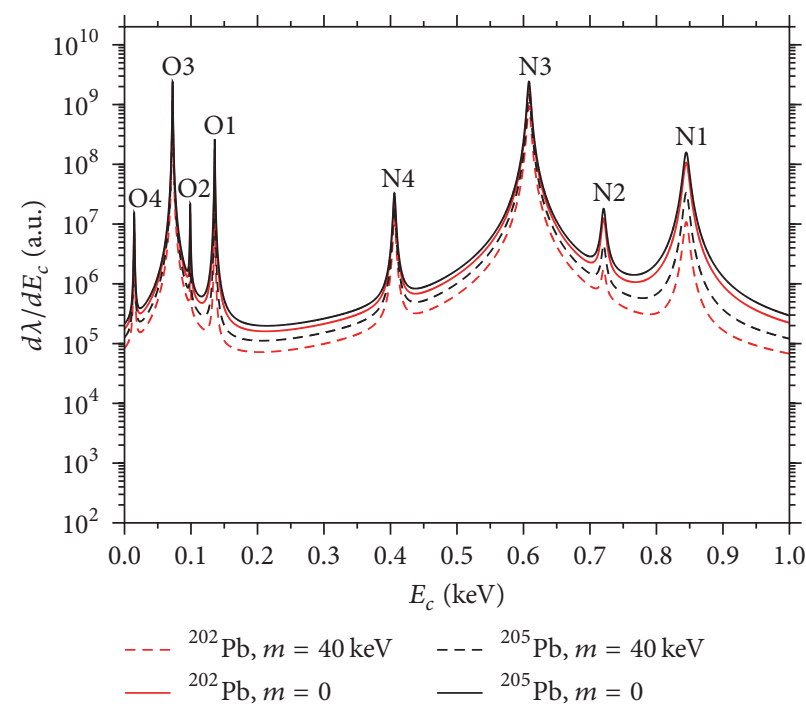

(b)

FIGURE 2: Calorimeter spectrum after electron capture in ${ }^{202} \mathrm{~Pb}$ (light curves) and in ${ }^{205} \mathrm{~Pb}$ (black curves) with emission of a light neutrino, $m_{l}=0$ (solid curves) and a heavy neutrino, $m_{h}=40 \mathrm{keV}$ (dashed curves). (a) Full spectrum, showing L and M capture peak labels. (b) Low energy region $\left(E_{c}=0-1 \mathrm{keV}\right)$, showing $\mathrm{N}$ and $\mathrm{O}$ capture peak labels.

\section{Results for Beta Decay Spectra}

In addition to electron capture, we also show here the effect of heavy neutrino emission in the electron spectrum of beta decays for one of the cases of most interest, Tritium. It has been studied in depth in previous works [84-86, 93], together with another process that has also drawn considerable theoretical end experimental attention, the beta decay of Rhenium 187 [37, 39-41, 84-86]. The beta decay of Tritium $\left({ }^{3} \mathrm{H} ; Z=1\right.$, $N=2)$ going to Helium $3\left({ }^{3} \mathrm{He} ; Z=2, N=1\right)$ is

$$
{ }^{3} \mathrm{H} \longrightarrow{ }^{3} \mathrm{He}+e^{-}+\bar{\nu}_{e}
$$

and has a Q-value of $18.59 \mathrm{keV}$ [92]. Both the initial and the final nuclear ground states have spin-parity $1 / 2^{+}$, and the transition is allowed with the electron and the antineutrino emitted in $s$-wave. The Karlsruhe Tritium Neutrino Experiment (KATRIN) [42-46] is currently studying this decay in order to determine the active neutrino mass and could also study the production of a heavy neutrino of a mass lower than $18 \mathrm{keV}$.

The differential decay rate with respect to the electron energy is given by

$$
\begin{aligned}
\frac{d \lambda}{d E_{e}}= & K_{\beta}\left(E_{e}^{2}-m_{e}^{2}\right)^{1 / 2}\left[\left(Q+m_{e}-E_{e}\right)^{2}-m_{\nu}^{2}\right]^{1 / 2} \\
& \cdot E_{e}\left(Q+m_{e}-E_{e}\right)
\end{aligned}
$$

where $K_{\beta}$ contains, among others, the weak interaction coupling, the nuclear matrix element, and the Fermi function.

As an illustration of the heavy neutrino effect in the Tritium beta decay, we plot in Figure 3 the differential decay rate from (24) and (5) using a maximal mixing with an unrealistic value of the mixing angle $\zeta=45^{\circ}$, to show the effect more distinctly in the scale of the figure. We have used heavy mass components with $m_{h}=2 \mathrm{keV}$ [26] (Figure 3(a)) and with $m_{h}=7 \mathrm{keV}[29,30]$ (Figure 3(b)). For comparison, the spectra without the heavy neutrino contribution $\left(\lambda^{h}=0\right)$ and without mixing $\left(\zeta=0^{\circ}\right)$ are also shown in this plot. The kink in the spectrum at $E_{e}-m_{e}=Q-m_{h}$ can be observed if the experimental relative error is lower than the size of the step, the latter being very small in realistic situations.

The effect of a heavy neutrino emission can be analyzed through the ratio between the heavy and the light neutrino contributions to the spectrum:

$$
\mathscr{R} \equiv \frac{d \lambda^{h} / d E_{e}}{d \lambda^{l} / d E_{e}} \tan ^{2} \zeta .
$$

The full differential decay rate is also related to the ratio $\mathscr{R}$ through

$$
\frac{d \lambda}{d E_{e}}=\frac{d \lambda^{l}}{d E_{e}}[1+\mathscr{R}] \cos ^{2} \zeta .
$$

In Figure 4 , we plot $\mathscr{R}$, the ratio of the heavy neutrino contribution over the light neutrino contribution to the decay rate, as a function of the momentum of the emitted electron for a heavy neutrino mass $m_{h}=2 \mathrm{keV}$ and different mixing angles: $\zeta=0.01^{\circ}, 0.005^{\circ}$, and $0.001^{\circ}$. The size of this ratio $\left(<10^{-7}\right)$ gives an idea of the difficulty of finding the kink in the spectrum due to the production of the heavy mass eigenstate. As can be seen in the figure, the ratio is different from zero and almost constant in the range $0 \leq p_{e}<\left(p_{e}\right)_{\max }$, where the maximum electron momentum is given by $\left(p_{e}\right)_{\max }=$ $\left[\left(Q-m_{h}\right)\left(Q-m_{h}+2 m_{e}\right)\right]^{1 / 2}$. For the heavy mass used in the figure, $\left(p_{e}\right)_{\max } \simeq 131.3 \mathrm{keV}$. The ratio $\mathscr{R}$ decreases as the mixing angle decreases, being approximately proportional to 


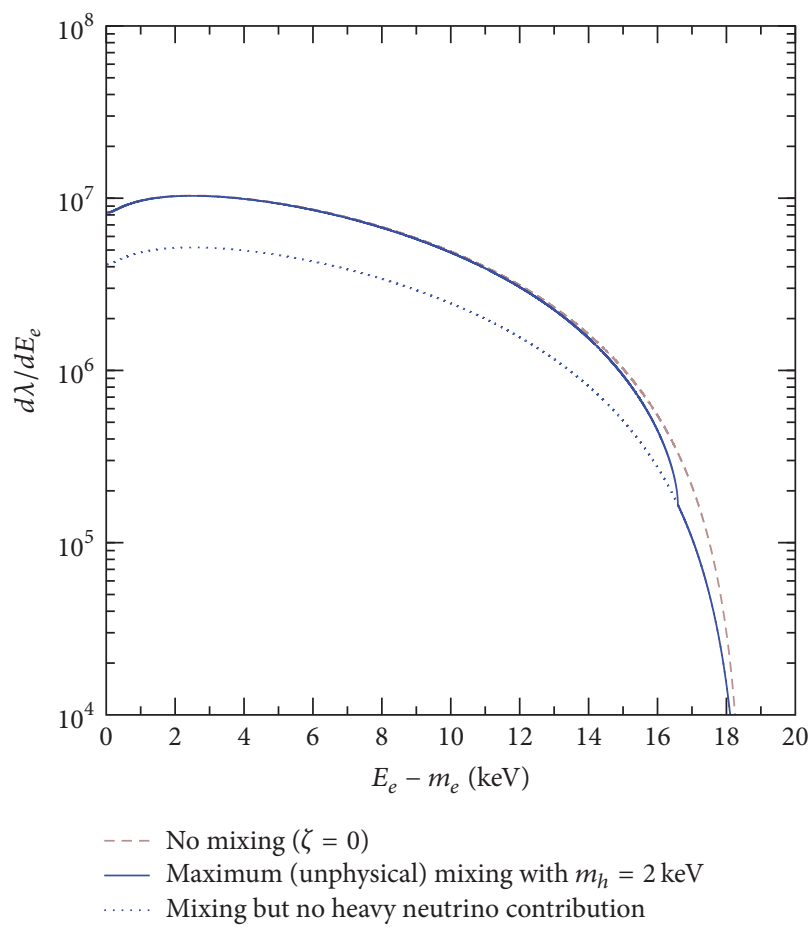

(a)

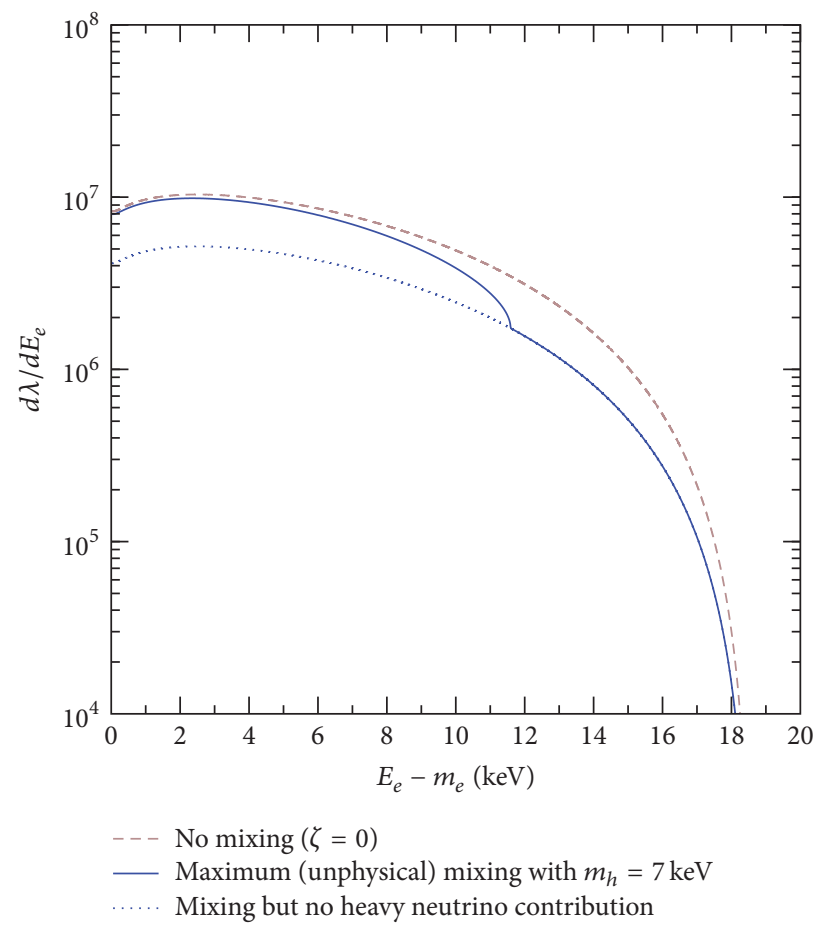

(b)

FIGURE 3: Electron spectrum of Tritium beta decay for a light-heavy neutrino mixing angle $\zeta=45^{\circ}$, shown just for illustration, and a heavy neutrino mass (solid curve) $m_{h}=2 \mathrm{keV}$ (a) and $m_{h}=7 \mathrm{keV}$ (b). The spectrum without heavy neutrino mass contribution (dotted curve) and without mixing (dashed curve) are also shown. We use the maximal unrealistic value $\zeta=45^{\circ}$ to make the effect visible in the scale of the figure. Realistic values of $\zeta<0.01^{\circ}$ result in a reduction of the difference between the dotted and the solid lines by more than seven orders of magnitude.

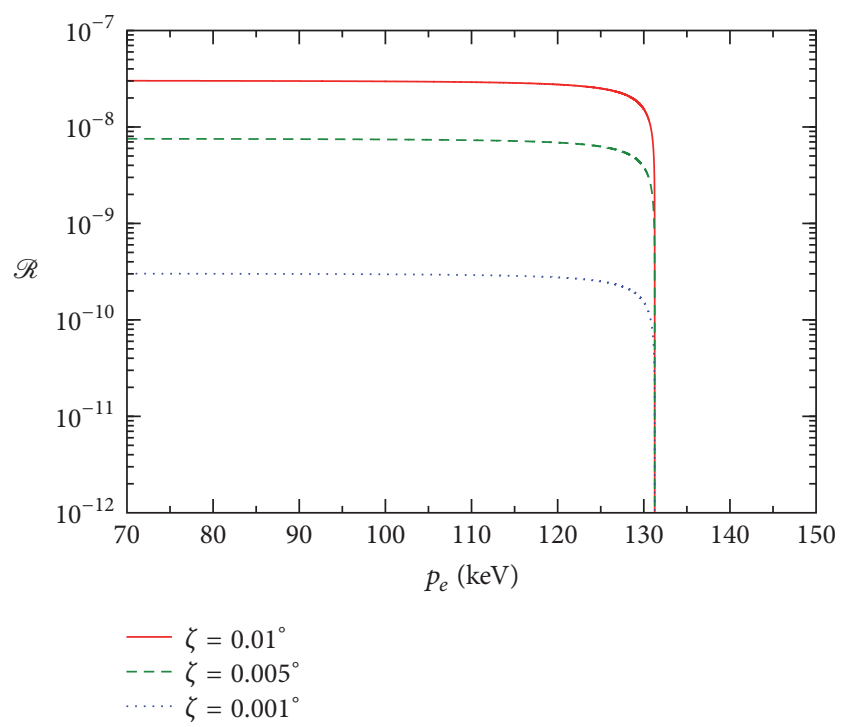

FIgURE 4: Ratio $\mathscr{R}$, defined in (25), for the Tritium beta decay, as a function of the electron momentum for a heavy neutrino mass $m_{h}=$ $2 \mathrm{keV}$ and different values of the mixing angle: $\zeta=0.01^{\circ}$ (solid line), $0.005^{\circ}$ (dashed line), and $0.001^{\circ}$ (dotted line).

$\zeta^{2}$. It also decreases for larger heavy neutrino masses $m_{h}$. A different ratio between the spectra with mixing and without mixing $\left(\mathscr{R}^{*}\right)$ has also been considered in [84-86, 93], related with $\mathscr{R}$ in (25) as

$$
\mathscr{R}^{*}=-\sin ^{2} \zeta+\mathscr{R} \cos ^{2} \zeta=\frac{d \lambda / d E_{e}}{d \lambda^{l} / d E_{e}}-1 .
$$

\section{Conclusions}

Signatures of hypothetical keV sterile neutrinos, which could be warm dark matter candidates (WDM), may be found in the spectra of ordinary weak nuclear decays. The electron spectrum in beta decay is cleaner for this purpose because it is a smooth curve, while the deexcitation spectrum after electron capture shows many peaks. On the other hand, in beta decay the possible excitation of the atoms or molecules is not disentangled while in electron capture the calorimeter collects all the energy independently of the excitation of the atom and of the deexcitation path, and one has higher statistics around the capture peaks.

Figures of electron spectrum for beta decay in ${ }^{3} \mathrm{H}$ as well as of calorimeter spectrum for electron capture in ${ }^{163} \mathrm{Ho}$ and ${ }^{202} \mathrm{~Pb}$ and ${ }^{205} \mathrm{~Pb}$ are given considering various values of the heavy neutrino mass $(2 \mathrm{keV}, 7 \mathrm{keV}$, and $40 \mathrm{keV})$ that may be experimentally probed. In both weak processes, the small value of the light-heavy neutrino mixing angle requires extremely high experimental precision and the use of sources with large stability to reduce systematic errors. This is why it 
is useful to consider relevant ratios between transition rates as first introduced in [84-87] to analyze the data. We consider two cases: the case of a single isotope, where one may use the ratio of accumulated number of events in different regions of the spectrum, which allows us to remove uncertainties related to the nuclear matrix element and to the values of overall constants. And in the case of two isotopes, we consider ratios of the above mentioned ratios that allow us to reduce uncertainties from atomic parameters. We give as well analytical expressions that can be used to obtain a good theoretical approximation to the experimental ratios ((19) to (21)).

Both the experimental measurement and the theoretical model must be accurate enough to detect differences in the expected versus the measured ratios of the order of the mixing angle squared, $\zeta^{2} \lesssim 10^{-8}$. This is also the size of the kink expected in the electron spectrum of beta decay, located at the limit of the region where the production of a heavy neutrino is energetically allowed. In order to identify this kink among the statistical fluctuations of the measured spectrum, the number of collected events must be larger than the inverse of the squared ratio $\mathscr{R}$ in (25).

Our results, particularly on the electron capture spectra of Lead isotopes that include for the first time all the possible peaks, may help in designing and analyzing future experiments to search for sterile neutrinos in the $\mathrm{keV}$ mass range. The use of the different types of ratios between number of events discussed here, for both electron capture and beta decay processes, may also help in planning the experiments by establishing the threshold of statistical and systematic uncertainties, required for the detection of sterile neutrinos of given mass and mixing, or to properly extract exclusion plots.

\section{Competing Interests}

The authors declare that they have no competing interests.

\section{Acknowledgments}

E. Moya de Guerra and O. Moreno acknowledge MINECO for partial financial support (FIS2014-51971-P) and IEMCSIC for hospitality. O. Moreno acknowledges support from a Marie Curie International Outgoing Fellowship within the European Union Seventh Framework Programme, under Grant Agreement PIOF-GA-2011-298364 (ELECTROWEAK). M. R. M. thanks N. G. Sánchez for correspondence and conversations.

\section{References}

[1] F. Zwicky, "Die Rotverschiebung von extragalaktischen Nebeln," Helvetica Physica Acta, vol. 6, pp. 110-127, 1933.

[2] M. Roos, "Astrophysical and cosmological probes of dark matter," Journal of Modern Physics, vol. 3, pp. 1152-1171, 2012.

[3] S. Dodelson, Modern Cosmology, Academic Press, New York, NY, USA, 2003.

[4] S. Weinberg, Cosmology, Oxford University Press, Oxford, UK, 2008.
[5] J. Lesgourgues and S. Pastor, "Neutrino mass from cosmology," Advances in High Energy Physics, vol. 2012, Article ID 608515, 34 pages, 2012.

[6] D. Boyanovsky, H. J. de Vega, and N. G. Sanchez, "Constraints on dark matter particles from theory, galaxy observations, and $N$-body simulations," Physical Review D, vol. 77, no. 4, Article ID 043518, 2008.

[7] D. Boyanovsky, H. J. de Vega, and N. G. Sanchez, "Dark matter transfer function: free streaming, particle statistics, and memory of gravitational clustering," Physical Review D, vol. 78, no. 6, Article ID 063546, 2008.

[8] C. Destri, H. J. de Vega, and N. G. Sanchez, "Warm dark matter primordial spectra and the onset of structure formation at redshift $z$," Physical Review D, vol. 88, no. 8, Article ID 083512, 2013.

[9] H. J. De Vega, P. Salucci, and N. G. Sánchez, "The mass of the dark matter particle: theory and galaxy observations," New Astronomy, vol. 17, no. 7, pp. 653-666, 2012.

[10] H. J. de Vega, P. Salucci, and N. G. Sanchez, "Observational rotation curves and density profiles versus the Thomas-Fermi galaxy structure theory," Monthly Notices of the Royal Astronomical Society, vol. 442, no. 3, pp. 2717-2727, 2014.

[11] H. J. de Vega and N. G. Sánchez, "Model-independent analysis of dark matter points to a particle mass at the keV scale," Monthly Notices of the Royal Astronomical Society, vol. 404, no. 2, pp. 885-894, 2010.

[12] H. J. de Vega and N. G. Sanchez, "Constant surface gravity and density profile of dark matter," International Journal of Modern Physics A, vol. 26, no. 6, pp. 1057-1072, 2011.

[13] H. J. de Vega and N. G. Sánchez, "Cosmological evolution of warm dark matter fluctuations. I. Efficient computational framework with Volterra integral equations," Physical Review D, vol. 85, no. 4, Article ID 043516, 20 pages, 2012.

[14] H. J. de Vega and N. G. Sanchez, "The dark matter distribution function and halo thermalization from the Eddington equation in galaxies," International Journal of Modern Physics A, vol. 31, no. 13, Article ID 1650073, 2016.

[15] N. Menci, S. Paduroiu, P. Salucci, N. Sánchez, and C. R. Watson, "lectures at the 20th Paris Cosmology Colloquium ChalongeHéctor de Vega," 2016, http://chalonge.obspm.fr/.

[16] E. W. Kolb and M. S. Turner, The Early Universe, Addison Wesley, Boston, Mass, USA, 1990.

[17] J. F. Navarro, C. S. Frenk, and S. D. M. White, "A universal density profile from hierarchical clustering," Astrophysical Journal Letters, vol. 490, no. 2, pp. 493-508, 1997.

[18] W. J. G. de Blok, “The core-cusp problem," Advances in Astronomy, vol. 2010, Article ID 789293, 14 pages, 2010.

[19] G. Gilmore, M. I. Wilkinson, R. F. G. Wyse et al., "The observed properties of dark matter on small spatial scales," Astrophysical Journal, vol. 663, no. 2, pp. 948-959, 2007.

[20] P. Salucci and Ch. Frigerio Martins, "The mass distribution in Spiral galaxies," EAS Publications Series, vol. 36, pp. 133-140, 2009.

[21] J. van Eymeren, C. Trachternach, B. S. Koribalski, and R.-J. Dettmar, "Non-circular motions and the cusp-core discrepancy in dwarf galaxies," Astronomy \& Astrophysics, vol. 505, no. 1, pp. $1-20,2009$.

[22] M. Walker and J. Peñarrubia, "A method for measuring (slopes of) the mass profiles of dwarf spheroidal galaxies," The Astrophysical Journal, vol. 742, no. 1, p. 20, 2011. 
[23] R. F. G. Wyse and G. Gilmore, "Observed properties of dark matter on small spatial scales," in Proceedings of the Proceedings of the International Astronomical Union Symposium (IUA '07), vol. 3, no. 244, pp. 44-52, Cardiff, UK, June 2007.

[24] A. Burkert, "The structure of dark matter halos in dwarf galaxies," Astrophysical Journal, vol. 447, no. 1, pp. L25-L28, 1995.

[25] C. Destri, H. J. de Vega, and N. G. Sánchez, "Quantum WDM fermions and gravitation determine the observed galaxy structures," Astroparticle Physics, vol. 46, pp. 14-22, 2013.

[26] N. Menci, N. G. Sanchez, M. Castellano, and A. Grazian, "Constraining the warm dark matter particle mass through ultra-deep UV luminosity functions at $Z=2$," The Astrophysical Journal, vol. 818, no. 1, article 90, 2016.

[27] E. Bulbul, M. Markevitch, A. Foster, R. K. Smith, M. Loewenstein, and S. W. Randall, "Detection of an unidentified emission line in the stacked X-ray spectrum of galaxy clusters," Astrophysical Journal, vol. 789, article 13, 2014.

[28] A. Boyarsky, O. Ruchayskiy, D. Iakubovskyi, and J. Franse, "Unidentified line in X-ray spectra of the andromeda galaxy and perseus galaxy cluster," Physical Review Letters, vol. 113, no. 25, Article ID 251301, 2014.

[29] K. N. Abazajian, "Resonantly produced $7 \mathrm{keV}$ sterile neutrino dark matter models and the properties of Milky Way satellites," Physical Review Letters, vol. 112, no. 16, Article ID 161303, 5 pages, 2014.

[30] N. Menci, A. Grazian, M. Castellano, and N. G. Sánchez, "Shock connectivity in the 2010 August and 2012 July solar energetic particle events inferred from observations and ENLIL modeling," Astrophysical Journal Letters, vol. 825, no. 1, article 1, 2016.

[31] A. D. Dolgov, "Neutrinos in cosmology," Physics Report, vol. 370, no. 4-5, pp. 333-535, 2002.

[32] A. Kusenko, "Sterile neutrinos: the dark side of the light fermions," Physics Reports, vol. 481, no. 1-2, pp. 1-28, 2009.

[33] B. Pontecorvo, "Neutrino experiments and the problem of conservation of leptonic charge," Soviet Physics-JETP, vol. 26, no. 5, pp. 984-988, 1968.

[34] A. Merle, "keV neutrino model building," International Journal of Modern Physics D, vol. 22, no. 10, Article ID 1330020, 2013.

[35] J. D. Vergados and T. S. Kosmas, "Searching for cold dark matter. A case of coexistence of supersymmetry and nuclear physics," Physics of Atomic Nuclei, vol. 61, p. 1066, 1998.

[36] E. Holmlund, M. Kortelainen, T. S. Kosmas, J. Suhonen, and J. Toivanen, "Microscopic calculation of the LSP detection rates for the ${ }^{71} \mathrm{Ga},{ }^{73} \mathrm{Ge}$ and ${ }^{127} \mathrm{I}$ dark-matter detectors," Physics Letters $B$, vol. 584, no. 1-2, pp. 31-39, 2004.

[37] R. Adhikari, M. Agostini, N. Anh Ky et al., "A white paper on $\mathrm{keV}$ sterile neutrino dark matter," https:/arxiv.org/abs/ 1602.04816 .

[38] G. Drexlin, V. Hannen, S. Mertens, and C. Weinheimer, "Current direct neutrino mass experiments," Advances in High Energy Physics, vol. 2013, Article ID 293986, 39 pages, 2013.

[39] MARE collaboration, http://mare.dfm.uninsubria.it/frontend/ exec.php.

[40] A. Nucciotti, "Neutrino mass calorimetric searches in the MARE experiment," https://arxiv.org/abs/1012.2290.

[41] A. Nucciotti, "On behalf of the MARE collaboration, lecture at the Workshop Chalonge Meudon," 2011, http://chalonge.obspm .fr/.

[42] KATRIN Collaboration, http://www.katrin.kit.edu.
[43] C. Weinheimer, "Direct determination of neutrino mass from tritium beta spectrum," https://arxiv.org/abs/0912.1619.

[44] C. Weinheimer, lecture at the 20th Paris Cosmology ChalongeHéctor de Vega Colloquium, 2016, http://chalonge.obspm.fr/.

[45] G. Drexlin lecture at the 19th Paris Cosmology Colloquium 2015.

[46] A. Huber, lecture at the Chalonge-de Vega Meudon Workshop, 2016, http://chalonge.obspm.fr/.

[47] C. Tully, lecture at the 19th Paris Cosmology Colloquium 2015.

[48] S. Betts, W. R. Blanchard, R. H. Carnevale et al., "Development of a relic neutrino detection experiment at PTOLEMY: princeton tritium observatory for light, early-universe, massiveneutrino yield," https://arxiv.org/abs/1307.4738.

[49] D. M. Asner, R. F. Bradley, L. de Viveiros et al., "Single-electron detection and spectroscopy via relativistic cyclotron radiation," Physical Review Letters, vol. 114, no. 16, Article ID 162501, 2015.

[50] P. C.-O. Ranitzsch, J.-P. Porst, S. Kempf et al., "Development of metallic magnetic calorimeters for high precision measurements of calorimetric 187Re and 163 Ho spectra," Journal of Low Temperature Physics, vol. 167, no. 5-6, pp. 1004-1014, 2012.

[51] C. Hassel, "Lecture at the International School of Astrophysics Daniel Chalonge-Héctor de Vega," 2015.

[52] L. Gastaldo, K. Blaum, A. Doerr et al., "The electron capture ${ }^{163}$ Ho experiment ECHo," Journal of Low Temperature Physics, vol. 176, no. 5, pp. 876-884, 2014.

[53] B. Alpert, M. Balata, D. Bennett et al., "The electron capture decay of ${ }^{163}$ Ho to measure the electron neutrino mass with subeV sensitivity," The European Physical Journal C, vol. 75, article 112, 2015.

[54] E. Fermi, "Radioattività prodotta da bombardamento di neutroni," Il Nuovo Cimento, vol. 11, no. 7, pp. 429-441, 1934.

[55] E. Fermi, "Versuch einer theorie der $\beta$-strahlen. I," Zeitschrift für Physik, vol. 88, no. 3, pp. 161-177, 1934.

[56] R. B. Firestone and V. S. Shirley, Eds., Table of Isotopes, John Wiley \& Sons, New York, NY, USA, 8th edition, 1999.

[57] W. Bambynek, H. Behrens, M. H. Chen et al., "Orbital electron capture by the nucleus," Reviews of Modern Physics, vol. 49, no. 1, pp. 77-221, 1977.

[58] J. L. Campbell and T. Papp, "Widths of the atomic K-N7 levels," Atomic Data and Nuclear Data Tables, vol. 77, no. 1, pp. 1-56, 2001.

[59] J. A. Bearden and A. F. Burr, "Reevaluation of X-ray atomic energy levels," Reviews of Modern Physics, vol. 39, no. 1, pp. 125142, 1967.

[60] M. Cardona and L. Ley, Eds., Photoemission in Solids I: General Principles, Springer, Berlin, Germany, 1978.

[61] J. C. Fuggle and N. Martensson, "Core-level binding energies in metals," Journal of Electron Spectroscopy and Related Phenomena, vol. 21, no. 3, pp. 275-281, 1980.

[62] T. Kosmas, H. Ejiri, and A. Hatzikoutelis, "Neutrino physics in the frontiers of intensities and very high sensitivities," Advances in High Energy Physics, vol. 2015, Article ID 806067, 3 pages, 2015.

[63] A. Giuliani and A. Poves, "Neutrinoless double-beta decay," Advances in High Energy Physics, vol. 2012, Article ID 857016, 38 pages, 2012.

[64] J.-U. Nabi and H. V. Klapdor-Kleingrothaus, "Weak interaction rates of sd-shell nuclei in stellar environments calculated in the proton-neutron quasiparticle random-phase approximation," Atomic Data and Nuclear Data Tables, vol. 71, no. 2, pp. 149-345, 1999. 
[65] J.-U. Nabi and H. V. Klapdor-Kleingrothaus, "Microscopic calculations of stellar weak interaction rates and energy losses for fp- and fpg-shell nuclei," Atomic Data and Nuclear Data Tables, vol. 88, no. 2, pp. 237-476, 2004.

[66] Y. Fukuda, T. Hayakawa, E. Ichihara et al., "Evidence for oscillation of atmospheric neutrinos," Physical Review Letters, vol. 81, no. 8, pp. 1562-1567, 1998.

[67] Q. R. Ahmad and SNO Collaboration, "Measurement of the rate of $v_{e}+d \rightarrow p+p+e^{-}$interactions produced by ${ }^{8} \mathrm{~B}$ solar neutrinos at the Sudbury Neutrino Observatory," Physical Review Letters, vol. 87, no. 7, Article ID 071301, 6 pages, 2001.

[68] Q. R. Ahmad, R. C. Allen, T. C. Andersen et al., "Direct evidence for neutrino flavor transformation from neutral-current interactions in the sudbury neutrino observatory," Physical Review Letters, vol. 89, no. 1, Article ID 011301, 2002.

[69] G. J. Feldman, J. Hartnell, and T. Kobayashi, "Long-baseline neutrino oscillation experiments," Advances in High Energy Physics, vol. 2013, Article ID 475749, 30 pages, 2013.

[70] S. T. Petcov, "The nature of massive neutrinos," Advances in High Energy Physics, vol. 2013, Article ID 852987, 20 pages, 2013.

[71] A. D. Dolgov, "Neutrinos in cosmology," Physics Report, vol. 370, no. 4-5, pp. 333-535, 2002.

[72] A. Kusenko, "Sterile neutrinos: the dark side of the light fermions," Physics Reports, vol. 48, no. 1-2, pp. 1-28, 2009.

[73] F. Munyaneza and P. L. Biermann, "Degenerate sterile neutrino dark matter in the cores of galaxies," Astronomy \& Astrophysics, vol. 458, no. 2, pp. L9-L12, 2006.

[74] D. Boyanovsky and C. M. Ho, "Sterile neutrino production via active-sterile oscillations: the quantum Zeno effect," Journal of High Energy Physics, vol. 2007, no. 7, p. 30, 2007.

[75] R. Shrock, "New tests for and bounds on neutrino masses and lepton mixing," Physics Letters B, vol. 96, no. 1-2, pp. 159-164, 1980.

[76] R. E. Shrock, "General theory of weak processes involving neutrinos. I. Leptonic pseudoscalar-meson decays, with associated tests for, and bounds on, neutrino masses and lepton mixing," Physical Review D, vol. 24, no. 5, pp. 1232-1274, 1981.

[77] R. E. Shrock, "Implications of neutrino masses and mixing for weak processes," in Proceedings of the Weak Interactions as Probes of Unification, vol. 72 of AIP Conference Proceedings, $\mathrm{p}$. 368, Blacksburg, Va, USA, 1981.

[78] J. M. Conrad, C. M. Ignarra, G. Karagiorgi, M. H. Shaevitz, and J. Spitz, "Sterile neutrino fits to short-baseline neutrino oscillation measurements," Advances in High Energy Physics, vol. 2013, Article ID 163897, 2013.

[79] A. Nucciotti, "The use of low temperature detectors for direct measurements of the mass of the electron neutrino," Advances in High Energy Physics, vol. 2016, Article ID 9153024, 41 pages, 2016.

[80] R. G. H. Robertson, "Examination of the calorimetric spectrum to determine the neutrino mass in low-energy electron capture decay," Physical Review C, vol. 91, no. 3, Article ID 035504, 2015.

[81] A. De Rújula, “Two old ways to measure the electron-neutrino mass," https://arxiv.org/abs/1305.4857.

[82] A. De Rújula, "An upper bound on the exceptional characteristics for Lusztig's character formula," https://arxiv.org/abs/0811 $.1674 \mathrm{v} 2$.

[83] A. Faessler and F. Šimkovic, "Can electron capture tell us the mass of the neutrino?" Physica Scripta, vol. 91, no. 4, Article ID 043007, 2016.
[84] H. J. de Vega, O. Moreno, E. Moya de Guerra, M. Ramón Medrano, and N. G. Sánchez, "Role of sterile neutrino warm dark matter in rhenium and tritium beta decays," Nuclear Physics B, vol. 866, no. 2, pp. 177-195, 2013.

[85] E. M. de Guerra, O. Moreno, P. Sarriguren, and M. Ramón Medrano, "Topics on nuclear structure with electroweak probes," Journal of Physics: Conference Series, vol. 366, Article ID 012011, 2012.

[86] J. M. Boillos, O. Moreno, and E. Moya de, "Active and sterile neutrino mass effects on beta decay spectra," in Proceedings of the La Rabida International Scientific Meeting on Nuclear Physics: Basic Concepts in Nuclear Physics: Theory, Experiments, and Applications, vol. 1541 of AIP Conference Proceedings, p. 167, La Rábida, Spain, September 2012.

[87] P. E. Filianin, K. Blaum, S. A. Eliseev et al., "On the keV sterile neutrino search in electron capture," Journal of Physics G: Nuclear and Particle Physics, vol. 41, no. 9, Article ID 095004, 2014.

[88] S. Eliseev, K. Blaum, M. Block et al., "Direct measurement of the mass difference of ${ }^{163} \mathrm{Ho}$ and ${ }^{163} \mathrm{Dy}$ solves the $Q$-value puzzle for the neutrino mass determination," Physical Review Letters, vol. 115, no. 6, Article ID 062501, 2015.

[89] A. De Rújula, "A new way to measure neutrino masses," Nuclear Physics, Section B, vol. 188, no. 3, pp. 414-458, 1981.

[90] M. Lusignoli and M. Vignati, "Relic antineutrino capture on ${ }^{163}$ Ho decaying nuclei," Physics Letters B, vol. 697, no. 1, pp. 11-14, 2011.

[91] A. Faessler, L. Gastaldo, and F. Šimkovic, "Electron capture in ${ }^{163}$ Ho, overlap plus exchange corrections and neutrino mass," Journal of Physics G: Nuclear and Particle Physics, vol. 42, no. 1, Article ID 015108, 2015.

[92] G. Audi, F. G. Kondev, M. Wang et al., "The Nubase2012 evaluation of nuclear properties," Chinese Physics C, vol. 36, no. 12, pp. 1157-1286, 2012.

[93] S. Mertens, "Sensitivity of next-generation tritium beta-decay experiments for keV-scale sterile neutrinos," JCAP, vol. 1502, no. 2, p. 20, 2015. 

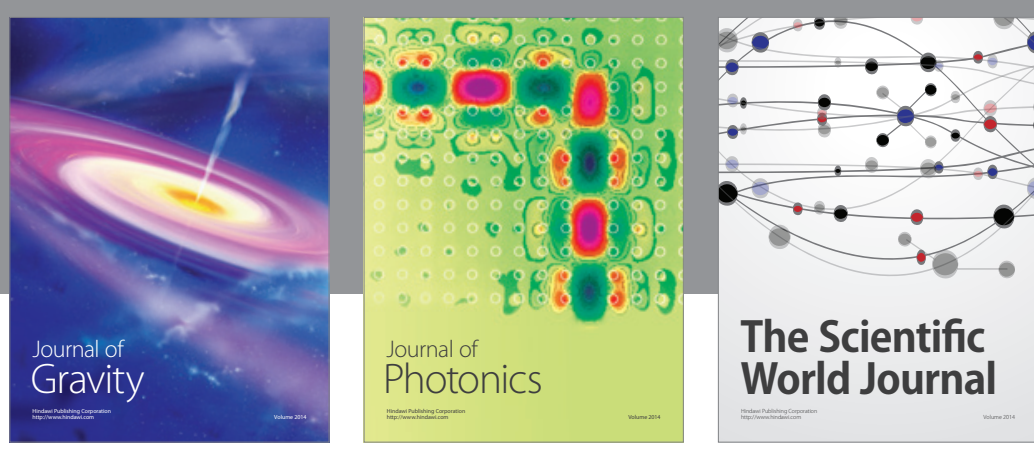

The Scientific World Journal
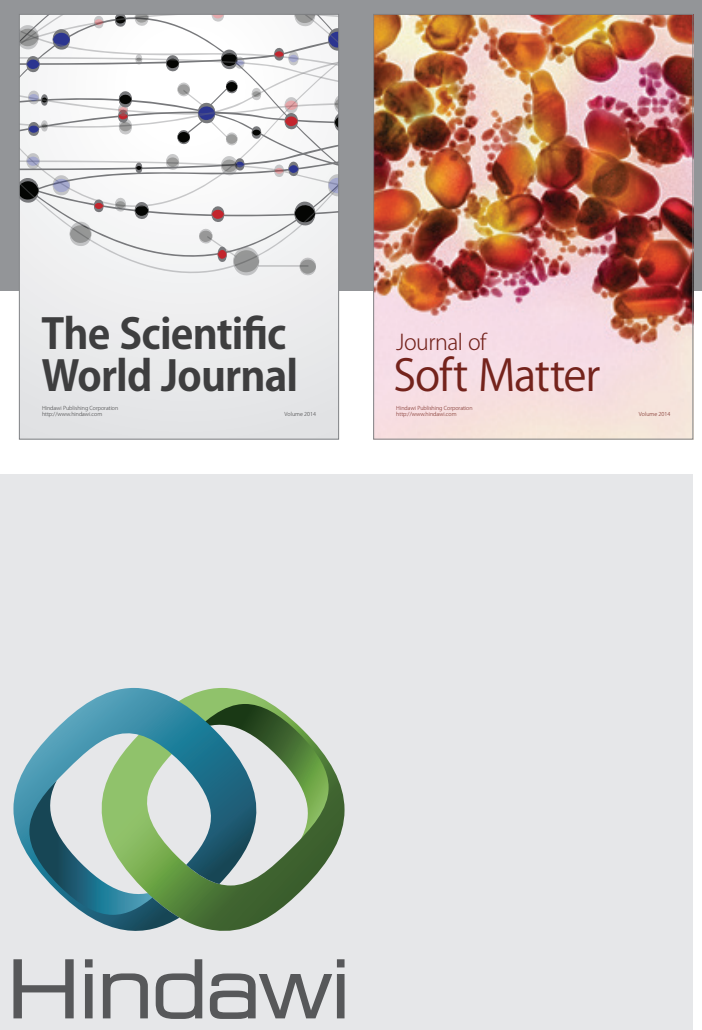

Submit your manuscripts at

http://www.hindawi.com

nternational Journal of

Statistical Mechanics
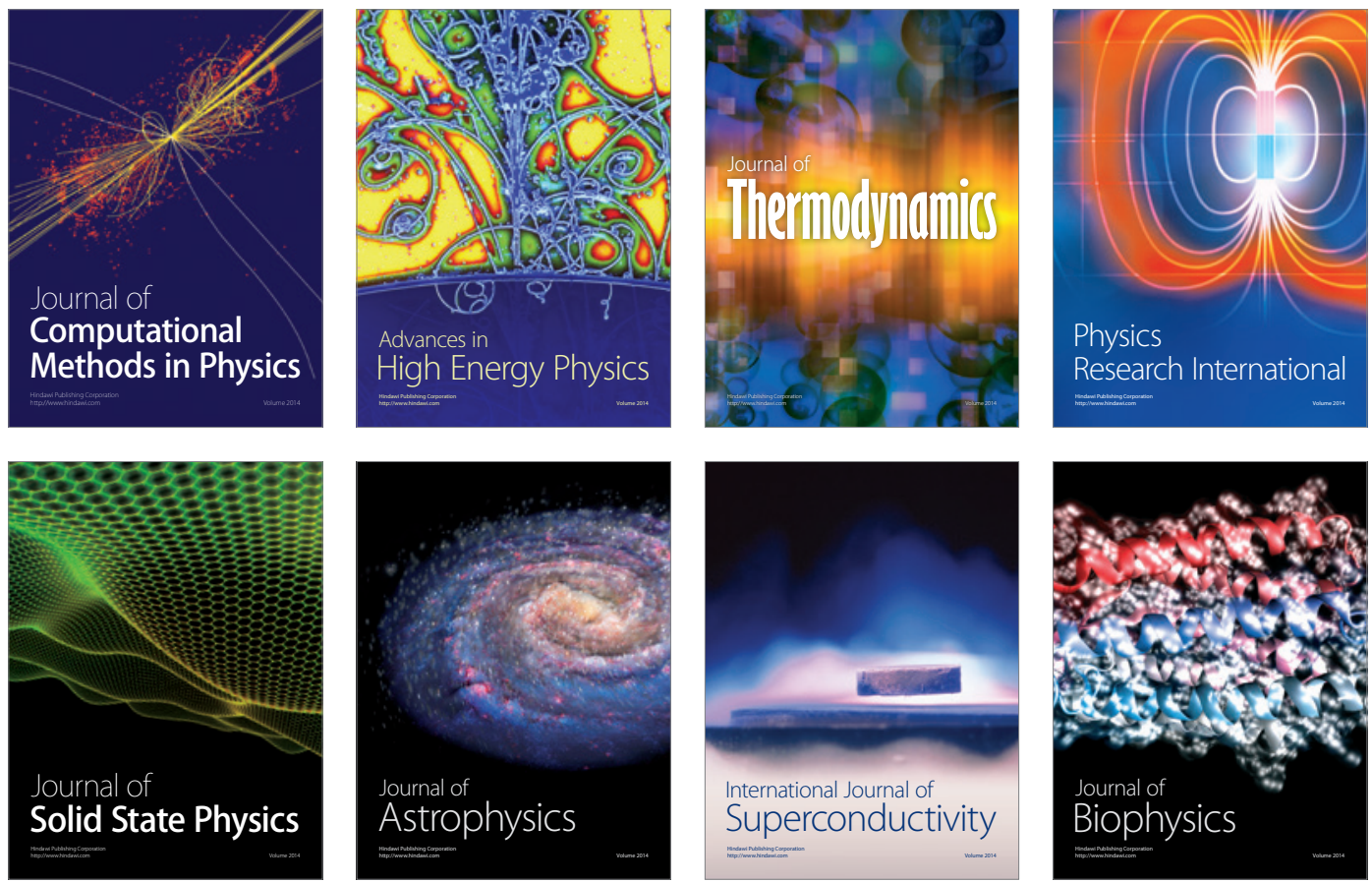
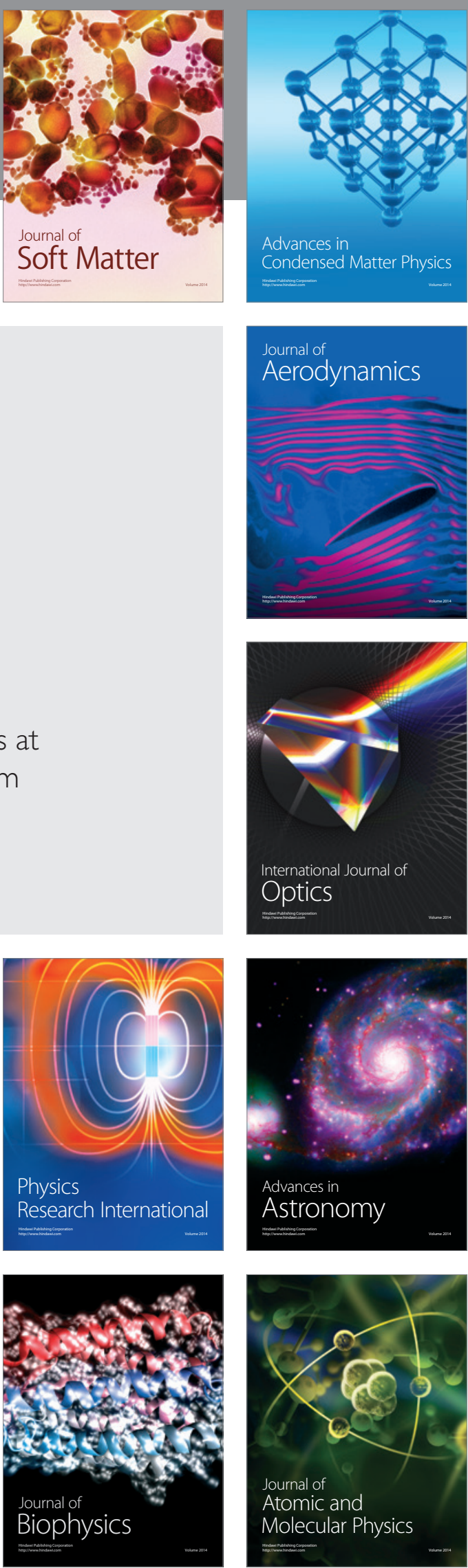